\title{
Influence of HLA-class II incompatibility between mother and fetus on the development and course of rheumatoid arthritis of the mother
} I E van der Horst-Bruinsma, R R P de Vries, P D M de Buck, P W van Schendel,
F C Breedveld, G M Th Schreuder, J M W Hazes

\begin{abstract}
Objective-To assess the relation between the course of rheumatoid arthritis (RA) during pregnancy or the onset of RA postpartum and DRB1, DQA1, and DQB1 incompatibilities between mother and child.

Methods-In 45 pregnancies of 33 RA patients the course of RA was related to the number of class II incompatibilities. Furthermore class II incompatibilities in 16 pregnancies followed by $\mathrm{RA}$ onset were compared with those in 87 control pregnancies.

Results-The risk of a favourable compared with an unfavourable course was $0.95,2.67$, and 2.38 in case of DRB1, DQA1, and DQB1 incompatibility respectively. DQA1 and DQB1 incompatibilities were seen more often in the 10 pregnancies followed by RA onset within three months than in control pregnancies (OR 8.02, 95\% CI 0.97, 66.06 and OR 8.79 95\% CI 1.07, 72.46 respectively).

Conclusions-DQA1 and DQB1 incompatibility between mother and child seems to have a favourable effect on the course of RA and may postpone the risk of RA onset during pregnancy.

(Ann Rheum Dis 1998;57:286-290)
\end{abstract}

Departments of Rheumatology I E van der

Horst-Bruinsma

P D M de Buck

$\mathrm{P}$ W van Schendel

F C Breedveld

J M W Hazes

and

Immunohaematology and Bloodbank

R R P de Vries

G M Th Schreuder

Leiden University Medical Centre,

Leiden, the

Netherlands

Correspondence to: Dr I E van der

Horst-Bruinsma, Leiden

University Hospital,

Department of

Rheumatology, C4-R, PO

Box 9600, 2300 RC Leiden,

the Netherlands.

Accepted for publication 17 March 1998
Pregnancy may provoke the onset of rheumatoid arthritis (RA) and influences the course of RA. ${ }^{1-3}$ During pregnancy amelioration of the disease is often observed and postpartum relapses may occur. ${ }^{4}$ Several hypotheses have been proposed for the role of hormones in the favourable effect of pregnancy on RA, but hormonal mechanisms cannot explain the differences in disease course between several pregnancies in one woman, unless the hormone levels differ in one woman. The different effects of pregnancies may be explained by an immunogenetic mechanism. Nelson et at $\bar{t}$ observed an increased number of HLA class II (DRB1, DQB1 and especially in DQA1 incompatibilities) between mother and child among women with diminished activity of RA during pregnancy. They proposed that this effect is caused by the influence of paternal DR and DQ molecules of the child on the maternal immune system during pregnancy.

Apart from immunogenetic mechanisms the sex of the child might influence the course of RA in the mother, for instance by the production of androgens in case of a male fetus. ${ }^{67}$
Pregnancy may, apart from influencing the course of RA, also play a part in the onset of RA. The risk of RA onset within three months post partum (OR 3.37-5.6) is increased and remains higher during the first year post partum (OR 2.6).$^{89}$ The risk of developing RA during pregnancy was found to be decreased (OR $0.3^{10}$ and OR $0.63^{9}$ ). The increased risk of RA onset in the postpartum period may also be explained in two ways: by hormonal mechanisms and by incompatibility in HLA class II antigens between mother and child. In both alternatives the disease onset is postponed because of the suppressive effect of pregnancy.

This study aimed to investigate (1) whether incompatibility in DRB1, DQA1, and DQB1 between mother and child is associated with a decrease in disease activity of RA during pregnancy and (2) whether HLA class II incompatibilities are associated with the risk of RA onset post partum in the mother. In addition the influence of the sex of the child on the onset and course of RA was studied.

\section{Methods}

PATIENTS

Women visiting the outpatient clinic of the Rheumatology Department of the University Hospital Leiden from February 1994 until March 1995 and who developed RA during their childbearing years were asked to participate in the study. The included patients fulfilled the 1987 ACR criteria for RA. ${ }^{11}$ Information about their pregnancies, miscarriages, the disease activity, and the medication used in the periods before, during, and after the pregnancies was obtained by patient interviews and by reviewing the medical records. Pregnancies with a RA onset within one year after delivery were considered as "onset related pregnancies" $(n=16)$. Pregnancies after the onset of RA were considered as "course pregnancies" $(n=33)$. The pregnancies of RA women who did not yet suffer from RA at that time nor had an RA onset within one year post partum were considered as "RA control pregnancies" $(n=16)$. The 71 "non-RA control pregnancies" were recruited from a study where the pregnancies of non-RA mothers of RA children were studied in the scope of the Non Inherited Maternal Antigen (NIMA) hypothesis that influences the risk of RA. ${ }^{12}$

Differences in HLA-DR and DQ antigens between mother and child for each pregnancy were related to the onset and course of RA in the mother. The study was designed as a case- 
control study in which the incompatibility of HLA-DR and DQ antigens between mother and child is the exposure variable. The time of onset of RA and the disease course during pregnancy are the outcome variables. The "cases" included either pregnancies related to the onset of RA (as "controls" pregnancies without an RA onset post partum were studied) or pregnancies during which the course of RA was studied (cases with a favourable course compared with cases with an unfavourable course). This implicates that one woman may contribute none or one "onset related pregnancy" and none or several "course pregnancies" depending on the onset of RA during her childbearing period.

To compare the number of incompatibilities in pregnancies followed by the onset of RA, the pregnancies of 87 women who did not develop RA at all were added to see whether this would show another pattern of incompatibilities in this larger group.

The study was approved by the medical ethical committee and informed consent was obtained from all subjects or from their parents.

\section{COURSE OF RA DURING PREGNANCY}

The course of RA during pregnancy was considered either favourable, unfavourable or unchanged. The incompatibility of HLA-DR and $\mathrm{DQ}$ in pregnancies with a favourable course were compared with those found in pregnancies with an unfavourable course. As this was a retrospective study, the information about the course of the disease during pregnancy was obtained from the medical charts and by a structural interview of the patients. The assessment of disease activity before, during, and after pregnancy is therefore not objective. It is known, however, that patients and doctors assessments are very sensitive and relevant instruments to measure disease activity ${ }^{13}$ and were therefore thought to be the most reasonable measurements to use in this study. The doctor's assessment of disease activity and the medication used three months before, during pregnancy, and in the year post partum were obtained by reviewing the medical record. The patient's assessment was obtained by an interview in which the patient could choose between remission, exacerbation or unchanged activity of RA in the before, during or after pregnancy periods. A favourable course was defined as decreased disease activity or remission of RA compared with the period before the pregnancy. In that case at least two of the following criteria had to be present: (1) remission or amelioration of disease during pregnancy according to the doctor's assessment, (2) same as in (1) but according to the patient's assessment, and (3) no increase of disease activity after the medication for RA had been stopped in the first trimester of the pregnancy.

An unfavourable course was defined as persistent disease activity or a flare of the disease during pregnancy. In that case at least two of the following criteria had to be present: (1) active disease during pregnancy according to the doctor's assessment, (2) same as in (1) but according to the patient's assessment, and (3) continuation of medication despite pregnancy. Cases with low disease activity before, during, and after the pregnancy were not included in this study, because the advantageous influence of the pregnancy on the course of RA could not be evaluated.

ONSET OF RA

The onset of RA was defined as the first symptoms of arthritis that finally resulted in the diagnosis of RA according to the ACR criteria. ${ }^{11}$

The DR and DQ incompatibilities of pregnancies followed by a RA onset within one year ("onset related pregnancies") were compared with (1) RA and non-RA control pregnancies, (2) pregnancies within the same women as the onset related pregnancies, but before the RA developed. Because the risk of RA onset is the highest within three months post partum ${ }^{10}$ a separate analysis was performed on pregnancies followed by RA onset within this period.

HLA TYPING

DNA was isolated from blood lymphocytes of the mothers and of the children included in the study. Generic DRB typing was performed with a polymerase chain reaction and biotin labelled sequence specific oligonucleotide (PCR-SSO) method as described previously. ${ }^{14}$ This method allows medium resolution DRB1 typing. DRB $1^{\star} 04$ subtyping (DRB $1^{\star} 0401-$ 0411) was performed by group specific amplification of DNA from all DRB1*04 positive women and hybridisation with relevant SSOs. ${ }^{15}$ In this way it was possible to distinguish homozygosity and heterozygosity for all $\mathrm{DRB} 1{ }^{\star} 04$ alleles and for the alleles known to express the shared epitope-that is, $\mathrm{DRB} 1{ }^{\star} 0101,{ }^{\star} 0102,{ }^{\star} 0401,{ }^{\star} 0404,{ }^{\star} 0405$, ${ }^{\star} 0408,{ }^{\star} 0410,{ }^{\star} 1001,{ }^{\star} 1402,{ }^{\star} 1406$. Generic DQA1 (0101-0104, 0201, 03, 0401, 0501, 0502 and 0601) and DQB1 (0501-0504, 0601-0609, 0201, 0202, 0301-0305, 0401, 0402) typing was performed with a PCR-SSO method as described previously. ${ }^{16}$

HLA class II incompatibilities between mother and child were defined from the perspective of the mother. Incompatibility was considered to be present if a HLA allele of the child differed from both maternal alleles.

\section{STATISTICS}

The differences in the number of incompatibilities in DRB1, DQA1, and DQB1 between the onset pregnancies, course pregnancies, and control pregnancies were tested with the $\chi^{2}$ test. The association between incompatibility and either favourable course of RA during pregnancy, or onset of RA post partum are represented as odds ratios with $95 \%$ confidence intervals. When the figure zero came up in the $\chi^{2}$ test a calculation was made adding 0.5 to all cells.

The comparison of incompatibilities in pregnancies within one woman was made considering the "onset related pregnancy" as reference case and the pregnancies before the onset 
Table 1 Course $\left(^{*}\right)$ of $R A$ during pregnancy in relation to incompatibility of $D R B 1, D Q A 1$, and $D Q B 1$ between mother and child

\begin{tabular}{clll}
\hline Incompatibilityt & $\begin{array}{c}\text { Favourable } \\
(n=21-23)\end{array}$ & $\begin{array}{l}\text { Unfavourable } \\
(n=5-6)\end{array}$ & OR $(95 \% \mathrm{CI})$ \\
\hline $\mathrm{DRB} 1+$ & 19 & 5 & $0.95(0.09,10.5)$ \\
- & 4 & 1 & $2.67(0.42,17.05)$ \\
$\mathrm{DQA1}+$ & 16 & 3 & \\
- & 6 & 3 & $2.38(0.17,33.00)$ \\
$\mathrm{DQB1}+$ & 19 & 4 & \\
- & 2 & 1 &
\end{tabular}

${ }^{\star}$ Course: disease activity of RA during pregnancy. Favourable: remission or amelioration of disease during pregnancy according to the criteria described in the methods. Unfavourable: active disease during pregnancy according to the criteria described in the methods. In 12 pregnancies the course of RA did not change and among these were $11 \mathrm{DRB} 1,10 \mathrm{DQA}$, and 7 DQB incompatibilities; they were not included in the calculations. †ncompatibility: when a child has an allele that is different from both maternal alleles. In some cases it was not possible to differentiate between incompatibility and compatibility according to the HLA data and they were left out, so the number of pregnancies vary for the different class II subtypes.

pregnancy as control pregnancies. All the pregnancies of one woman were considered as a matched set. The incompatibility was the exposure.

\section{Results}

Of the 362 patients interviewed 62 women were eligible for the study and agreed to participate. These 62 women gave birth to 160 children of which 142 children participated in the study. The women had a mean number of children of 2.6 and a mean age of RA onset of 33 years. Forty five $(72 \%)$ RA patients were rheumatoid factor positive, $44(71 \%)$ had erosions, and $11(18 \%)$ nodules. Alleles expressing the shared epitope were found in a double dose in 13 women $(21 \%)$, a single dose in 37 women $(60 \%)$, and none in 14 women $(22 \%)$.

COURSE OF RA

The course of RA could be studied in only 45 pregnancies of 20 women, because the other women have not yet developed RA in their childbearing period. The pregnancies of these women served as controls. Twelve pregnancies were excluded because of low disease activity before the pregnancy and no change of RA during or after the pregnancy. None of the women showed a favourable course during one pregnancy and an unfavourable course in the other pregnancy. A combination of an unchanged low level of disease activity in one

Table 2 HLA incompatibility in onset pregnancies and control pregnancies

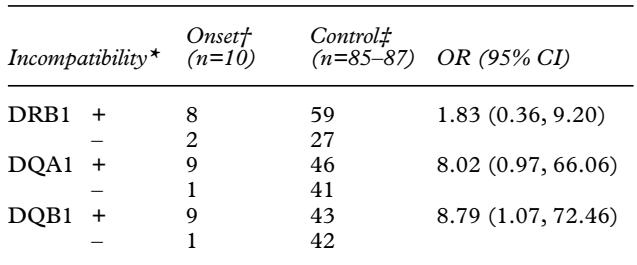

^Incompatibility: when a child has an allele that is different from both maternal alleles. †Onset pregnancy: RA developed within three months after pregnancy. $\ddagger$ Control pregnancy: pregnancies of women who did not develop RA within one year after pregnancy. In some cases it was not possible to differentiate between incompatibility and compatibility according to the HLA data and they were left out, so the number of pregnancies vary for the different class II subtypes. pregnancy and a favourable course in one or more other pregnancies in the same woman was observed in three women. Eventually, 33 pregnancies showed either a favourable $(n=27)$ or an unfavourable course of RA $(n=6)$ (table 1). It was not possible to differentiate between incompatibility and compatibility according to the HLA data in seven pregnancies according to DQB1 (six with a favourable and one with an unfavourable course) and in five cases according to the DQA1 and four to the DRB1 typing (all with a favourable course). The odds ratio (OR) of a favourable course of RA during pregnancy in case of DRB1 incompatibility compared with DRB1 compatibility was 0.95 (95\% CI 0.09, 10.5). DQA1 and DQB1 incompatibility occurred more often in pregnancies with a favourable course, OR 2.67 (95\% CI $0.42,17.05)$ and 2.38 (95\% CI 0.17 , 33.00) respectively (table 1 ).

ONSET OF RA

Sixteen women developed RA within one year after delivery. The HLA incompatibilities of these pregnancies were compared with the HLA incompatibilities of the RA and non-RA control pregnancies. It was not possible to differentiate between incompatibility and compatibility according to the HLA data in eight pregnancies in DRB1, seven DQA1, and in nine DQB1 typings, all from the control group. RA onset post partum was slightly increased in case of DRB1 incompatibility (OR 1.37, 95\% CI $0.41,4.65$ ), but more in case of DQA1 (OR $3.86,95 \%$ CI $1.03,14.52)$ and DQB1 incompatibility (OR 4.23, 95\% CI 1.12, 15.9).

By restricting the analysis to the 10 women who experienced RA onset within three months post partum, the risk for DRB1 incompatibility remained similar (OR 1.83 $95 \%$ CI $0.36,9.20)$ but increased to $8.02(95 \%$ CI $0.97,66.06)$ and 8.79 (95\% CI 1.07, 72.46) in case of DQA1 and DQB1 incompatibility respectively (table 2). If RA control and non-RA control pregnancies were considered separately, similar results were obtained. In the matched analysis all pregnancies within one woman were considered as a matched set. The conditional logistic regression analysis using the statistical program Egret did not result in a defined odds ratio, because of the small numbers. Therefore the crude data are also presented (table 3).

When RA onset related pregnancies are restricted to the ones followed by RA within three months post partum all six pregnancies show incompatibility in DQA 1, DQB1, and DRB1. Also every pregnancy before these "RA onset related pregnancies within three month" show incompatibilities of these three class II types except for one case of DQB1 compatibility.

In case of a male child the risk of developing RA post partum was (not significantly) higher than in case of a female child (OR 1.85, 95\% CI $0.48,7.18$ ). The risk of an unfavourable course of RA during pregnancy was (not significantly) increased by a male child compared with a female child (OR 1.6, 95\% CI $0.25,10.2)$. 
Table 3 HLA incompatibility of the $R A$ onset related pregnancy and the previous pregnancies of these women

\begin{tabular}{|c|c|c|c|c|c|}
\hline \multirow[b]{2}{*}{ Woman } & \multirow[b]{2}{*}{ Pregnancies } & \multicolumn{4}{|c|}{ Incompatibility } \\
\hline & & $D Q A 1$ & $D Q B 1$ & $D R B 1$ & $\begin{array}{l}R A \text { onset } \\
\text { postpartum }\end{array}$ \\
\hline \multirow[t]{3}{*}{1} & 1 & 1 & 1 & 1 & no \\
\hline & 2 & 1 & 1 & 1 & no \\
\hline & 3 & 0 & 0 & 0 & yes \\
\hline \multirow[t]{2}{*}{2} & 1 & 1 & 0 & 1 & no \\
\hline & 2 & 1 & 1 & 1 & yes \\
\hline \multirow[t]{2}{*}{3} & 1 & 1 & 1 & 1 & no \\
\hline & 2 & 1 & 1 & 1 & yes \\
\hline \multirow[t]{2}{*}{4} & 1 & 1 & 1 & 1 & no \\
\hline & 2 & 1 & 1 & 0 & yes \\
\hline \multirow[t]{3}{*}{5} & 1 & 1 & 1 & 1 & no \\
\hline & 2 & 1 & 1 & 1 & no \\
\hline & 3 & 1 & 1 & 1 & yes \\
\hline \multirow[t]{2}{*}{6} & 1 & 1 & 1 & 1 & no \\
\hline & 2 & 1 & 1 & 1 & yes \\
\hline \multirow[t]{2}{*}{7} & 1 & 1 & 1 & 1 & no \\
\hline & 2 & 1 & 1 & 1 & yes \\
\hline \multirow[t]{4}{*}{8} & 1 & 0 & 0 & 1 & no \\
\hline & 2 & 1 & 1 & 1 & no \\
\hline & 3 & 0 & 0 & 1 & no \\
\hline & 4 & 0 & 0 & 1 & yes \\
\hline \multirow[t]{2}{*}{9} & 1 & 1 & 1 & 1 & no \\
\hline & 2 & 1 & 1 & 1 & yes \\
\hline \multirow[t]{2}{*}{10} & 1 & 1 & 1 & 1 & no \\
\hline & 2 & 1 & 1 & 1 & yes \\
\hline
\end{tabular}

$0=$ Compatibility, $1=$ incompatibility.

\section{Discussion}

DQA1 and DQB1 incompatibility between mother and child was found more often in pregnancies with a favourable course of RA than in those with an unfavourable course. There was no relation between DRB1 incompatibilities and the course of RA. In addition pregnancies with a postpartum onset of RA within three months also showed an increased number of DQ incompatibilities and no effect of DRB1 incompatibilities when compared with pregnancies of women who did not develop RA in their childbearing years.

Despite a major effort to include as many women as possible, the number of women that could be studied remained low as in most women RA started several years after child birth.

COURSE OF RA DURING PREGNANCY

Many theories have been proposed to explain why RA ameliorates during pregnancy and often exacerbates post partum. Hormones like oestrogen, ${ }^{17}{ }^{18}$ progesterone, corticosteroid, and prolactin ${ }^{19}{ }^{20}$ have been put forward. Also it has been postulated that androgens have an anti-inflammatory effect on $\mathrm{RA}^{2122}$ and according to some even the small amounts produced by a male fetus might ameliorate RA during pregnancy. $^{67}$

An explanation of the ameliorating effect of pregnancy in RA by androgen concentrations as result of sex differences of the children could not be confirmed in this study.

The hormonal theories cannot explain, however, why the course of RA may vary during different pregnancies. The fact that every pregnancy results in a different set of class II incompatibilities that has a different effect on disease activity of RA may offer an attractive explanation. Leucocytes or shed soluble HLA molecules of the child may enter the maternal circulation and influence the maternal immune response in a still undefined way. This is illustrated by the lymphocytotoxic anti-HLA ABC and DR antibodies found in $9 \%$ of the women after their first and $18 \%$ after subsequent pregnancies. ${ }^{23}$

This study shows a clear trend towards a disease suppressing effect of DQA1 and DQB1 incompatibility during pregnancy, confirming the study of Nelson. ${ }^{5}$ In line with this previous study $^{5}$ there seems to be a greater influence of DQ than of DR (mis)matching on the course of RA. The immunogenetic methods of both studies were compared in detail and showed no differences in DNA typing techniques nor in interpretation of incompatibility. Compared with Nelson et $a \bar{l}$ we observed more often a favourable course, which fits with the fact that we observed more often DR and DQ incompatibilities. The present data support the notion that DQ molecules may be involved in the predisposition to and course of RA. The influence of DQ (in particular DQ7) on disease severity of RA has been shown in a study comparing mild with severe forms of RA in DR4 positive RA patients. ${ }^{24}$ Based on evidence in transgenic mouse models Zanelli ${ }^{25}$ suggested that DQ rather than DR alleles predispose to RA.

\section{ONSET OF RA IN RELATION TO PREGNANCY}

The onset of RA in women during their fertile lifetime was found to be related in $23-30 \%$ of the women to a pregnancy. ${ }^{1026}$ In $15 \%$ of the women the disease develops within three months post partum and in 5\% between three months and one year post partum. ${ }^{8-1026-29}$

There is much debate about the mechanism that explains the increased risk of RA onset within three months post partum. One explanation is that hormonal or immunological mechanisms may play a part in postponing the onset of RA by suppressing disease activity during pregnancy. The relatively low incidence of RA onset during pregnancy ${ }^{9}{ }^{10}$ supports this theory. In accordance with pregnancies associated with a favourable course, pregnancies followed by RA onset also showed an increased number of DQA and DQB incompatibilities compared with controls. Therefore we interpret this as being the result of an extra suppressive effect during pregnancy of an ongoing RA that became manifest within three months post partum. The results of the matched analysis that showed that the majority of the women with a RA onset within three months post partum already had been exposed to an incompatibility before, can be explained by the absence of other susceptibility factors during the previous pregnancies. However, the numbers in that analysis were too small to challenge this hypothesis or to deserve an additional explanation.

To our knowledge the topic of HLA incompatibility, inclusive DQA1 and DQB1, between mother and child and the onset of RA has not been studied before. Brennan et $a l^{\beta 0}{ }^{31}$ found no association between fetally inherited DRB1 paternal antigens and the onset of RA within the mother, but these investigators did not look into HLA incompatibilities between mother 
and child by comparing each pregnancy of a woman.

In conclusion, QB1 and DQA1 incompatibilities between mother and child were found more often in pregnancies with a favourable course compared with those with an unfavourable course. Also DQA1 and DQB1 incompatibilities were seen more often in pregnancies followed by RA onset than among control pregnancies, which suggests that DQ incompatibility might postpone the onset of RA. These results support the suggestion put forward by others that DQ antigens may play a more prominent part in RA than has been generally appreciated until now.

1 Hench PS. The ameliorating effect of pregnancy on chronic atrophic (infectiuos rheumatoid) arthritis, fibrositis and intermittent hydrarthrosis. Mayo Clin Proc 1938;13:161-7.

2 Cecere FA, Persellin RH. The interaction of pregnancy and the rheumatic diseases. Clinics in Rheumatic Diseases 1981;7:747-51.

3 Neely NT, Persellin RH. Activity of rheumatoid arthritis during pregnancy. Tex Med 1977;73:59-63.

4 Ostensen M. Treatment with immunosuppressive and disease modifying drugs during pregnancy and lactation. disease modifying drugs during pregnan

5 Nelson JL, Hughes KA, Smith AG, Nisperos BB, BranNelson JL, Hughes KA, Smith AG, Nisperos BB, Bran-
chaud AM, Hansen JA. Maternal-fetal disparity in HLA chaud AM, Hansen JA. Maternal-fetal disparity in HLA tion of rheumatoid arthritis. N Engl J Med 1993;329:46671 .

6 Cutolo M, Accardo S. Sex hormones, HLA and rheumatoid arthritis. Clin Exp Rheumatol 1991;9:641-6.

7 James WH. Rheumatoid arthritis, the contraceptive pill, and androgens. Ann Rheum Dis 1993;52:470-4.

8 Silman AJ. Parity status and the development of rheumatoid arthritis. Am J Reprod Immunol 1992;28:228-30.

9 Lansink M, de Boer A, Dijkmans BA, Vandenbroucke JP, Hazes JM. The onset of rheumatoid arthritis in relation to pregnanc

10 Silman AJ, Kay A, Brennan P. Timing of pregnancy in relation to the onset of rheumatoid arthritis. Arthritis Rheum 1992;35:152-5.

11 Arnett FC, Edworthy SM, Bloch DA, McShane DJ, Fries $\mathrm{JF}$, Cooper NS, et al. The American Rheumatism Associa$\mathrm{JF}$, Cooper NS, et al. The American Rheumatism Associa-
tion 1987 revised criteria for the classification of rheumation 1987 revised criteria for the classification of

12 Wolde ten S, Breedveld FC, Vries de RRP, D'Amaro J, Rubenstein P, Schreuder GMTh, et al. Influence of non-inherited maternal HLA antigens on occurrence of rheumatoid arthritis. Lancet 1993;341:200-2.

13 Tugwell P, Boers M. OMERACT conference on outcome measures in rheumatoid arthritis clinical trials. J Rheumatol 1993;20:528-9.
14 Verduyn W, Doxiadis IIN, Anholts J, Drabbels JJM, Naipal A, D'Amaro J, et al. Biotinylated DRB sequence-specific oligonucleotides; comparison to serologic HLA-DR typing of organ donors in Eurotransplant. Hum Immunol 1993;37:59-67.

15 Gao X, Olsen NJ, Pincus T, Stastny P. HLA-DR alleles with naturally occurring amino acid substitutions and risk for development of rheumatoid arthritis. Arthritis Rheum 1990;33:939-46.

16 Giphart MJ, Roep BO, D'Amaro J. Relative contribution of HLA-DQA- and DQB-alleles to predisposition to insulin dependent diabetes mellitus. Hum Immunol 1992;34: $142-6$.

17 Da Silva JA, Hall GM. The effects of gender and sex hormones on outcome in rheumatoid arthritis. Baillieres Clin Rheumatol 1992;6:196-219.

18 Mattsson R, Mattsson A, Holmdahl R, Whyte A, Rook GA. Maintained pregnancy levels of oestrogen afford complete protection from post-partum exacerbation of collagenprotection from post-partum exacerbation of coll

19 Brennan P, Silman A. Breast-feeding and the onset of rheumatoid arthritis. Arthritis Rheum 1994;37:808-13

20 Chikanza IC, Petrou P, Chrousos G, Kingsley G, Panayi GS. Excessive and dysregulated secretion of prolactin in rheumatoid arthritis: immunopathogenic and therapeutic implications. Br J Rheumatol 1993;32:445-8.

21 Pritchard $\mathrm{MH}$. An exmanination of the role of female hormones and pregnancy as risk factors for rheumatoid arthritis, using a male population as control group. $\mathrm{Br} \mathrm{J}$ Rheumatol 1992;31:395-9.

22 Masi AT. Incidence of rheumatoid arthritis: do the observed age-sex interaction patterns support a role of androgenicanabolic steroid deficiency in its pathogenesis? $\mathrm{Br} \mathrm{J}$ Rheumatol 1994;33:697-701.

23 Morin-Papunen L, Tilikainen A, Hartikainen-Sorri AL. Maternal HLA immunization during pregnancy: presence of anti HLA antibodies in half of multigravidous women. Medical Biology 1984;62:323-5.

24 Singal DP, Green D, Reid B, Gladman DD, Buchanan WW. HLA-D region genes and rheumatoid arthritis (RA): importance of DR and DQ genes in conferring susceptibility to RA [see comments]. Ann Rheum Dis 1992;51:23-8.

25 Zanelli E, Gonzalez-Gay MA, David CS. Could HLADRB1 be the protective locus in rheumatoid arthritis? Immunol Today 1995;16:274-8.

26 Oka M. Effect of pregnancy on the onset and course of rheumatoid arthritis. Ann Rheum Dis 1953;1:227-9.

27 Hazes JM, Dijkmans BA, Vandenbroucke JP, de Vries RR, Cats A. Pregnancy and the risk of developing rheumatoid arthritis. Arthritis Rheum 1990;33:1770-5.

28 Silman AJ. Is pregnancy a risk factor in the causation of rheumatoid arthritis? Ann Rheum Dis 1986;45:1031-4.

29 Ostensen M, Husby G. A prospective clinical study of the effect of pregnancy on rheumatoid arthritis and ankylosing spondylitis. Arthritis Rheum 1983;26:1155-9.

30 Brennan P, Payton A, Ollier B, Silman A. Maternal exposure to paternal HLA does not explain the postpartum increase in rheumatoid arthritis. Genet Epidemiol 1996;13:411-18.

31 Brennan P, Silman AJ, Payton A, Bamber S, Ollier WER. Foetally inherited paternal HLA does not explain post partum susceptibility to rheumatoid arthritis in the mother. $\mathrm{Br}$ J Rheumatol 1995;34:142. 